\title{
The LUCID-2 detector
}

\author{
A. Sbrizzi ${ }^{1, a}$, on behalf of the ATLAS Collaboration \\ ${ }^{1}$ INFN Bologna
}

\begin{abstract}
The LUCID-2 detector is the main online and offline luminosity monitor of the ATLAS experiment. It is a compact Cherenkov detector made of photomultipliers with a $10 \mathrm{~mm}$ diameter quartz window acting as the Cherenkov medium. In order to provide a reliable luminosity measurement during years of data-taking, the LUCID-2 detector can use 104 different luminosity algorithms for each of the LHC colliding bunches. The detector stability is kept at a percent level thanks to an innovative monitoring system based on radioactive $\mathrm{Bi}-207$ sources deposited on the photomultipliers quartz window. Combining the LUCID-2 measurement with those of other four ATLAS subdetectors, the luminosity has been estimated with a total uncertainty of $2.1 \%$ in 2015 and $2.2 \%$ in 2016 .
\end{abstract}

\footnotetext{
a e-mail: Antonio.Sbrizzi@ cern.ch
} 


\section{Introduction}

The luminosity is a key parameter for colliders in high energy particle physics. Online luminosity monitoring is required for the operation of the collider, while the total uncertainty on the luminosity is often limiting the accuracy of cross section measurements. The LUCID detector [1] and the associated read-out electronics were upgraded to provide the measurement of luminosity during LHC Run 2 with up to 80 simultaneous pp collisions per bunch crossing at $13 \mathrm{TeV}$ center-of-mass energy and $25 \mathrm{~ns}$ bunch-spacing.

\section{The LUCID-2 detector}

The LUCID-2 detector [2] is a compact Cherenkov detector made of photomultipliers with a $10 \mathrm{~mm}$ diameter quartz window acting as the Cherenkov medium (Hamamatsu R760). Two modules are installed symmetrically around the pp collision point at a longitudinal distance of $17 \mathrm{~m}$. The radial distance of the photomultipliers from the beamline is $12 \mathrm{~cm}$. In collaboration with Hamamatsu, the acceptance of some photomultipliers was reduced by depositing a ring of Aluminium between the quartz window and the photocathode in order to cope with the high particle density close to the beampipe. A special subdetector is equipped with quartz fibers acting simultaneously as Cherenkov medium and light guide towards photomultipliers situated $1.5 \mathrm{~m}$ away in a shielded location. Figure 1 shows one of the two LUCID-2 modules, each made of 20 photomultipliers.

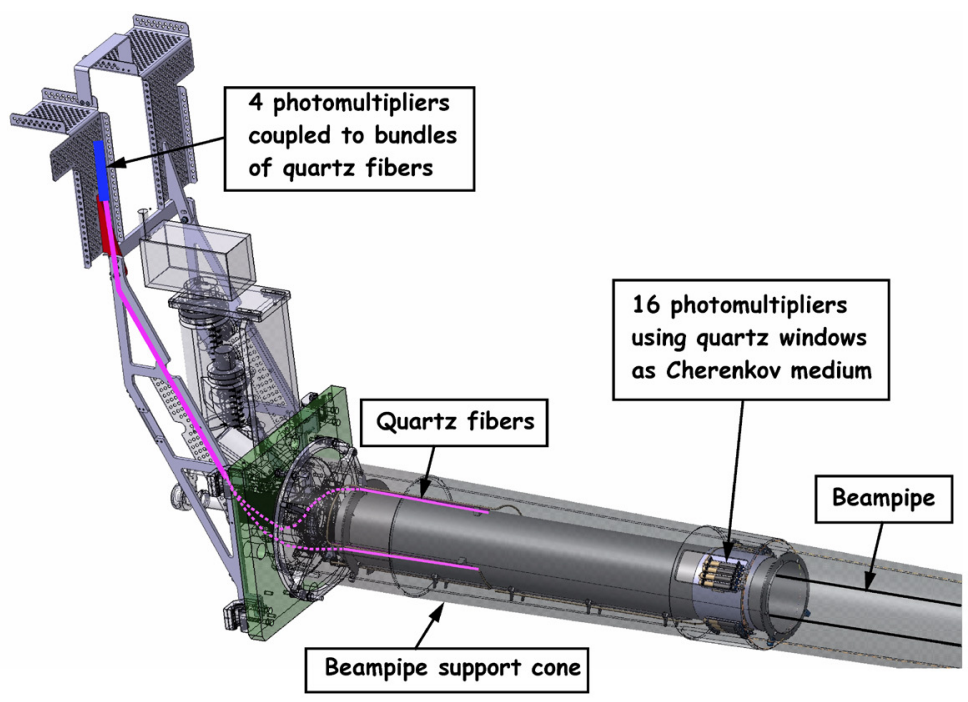

Figure 1. Schematic view of one LUCID-2 module. The 16 photomultipliers installed around the beampipe are divided into 4 subdetectors. An additional subdetector made of 4 photomultipliers is located far away from the beamline in a shielded location and is used to readout Cherenkov light signals produced in quartz fibers.

A total of $40(20+20)$ photomultipliers is divided into 5 subdetectors. The main detector $(4+4)$ is for ATLAS luminosity monitoring. A spare detector (4+4) and the one with lower acceptance (4+4) are used as back-up detectors and provide independent luminosity measurements for cross-checks. An additional spare subdetector (4+4) is kept turned off. The fiber detector (4+4) is a prototype for the upgraded LHC accelerator with higher luminosity (HL-LHC). 
Custom VME boards called LUCRODs are placed at a distance of about $15 \mathrm{~m}$ from the detector to perform signal digitisation and fast processing. The sampling rate of the 12-bits flash ADCs is $320 \mathrm{MS} / \mathrm{s}$, the optical data transfer is $1.3 \mathrm{~Gb} / \mathrm{s}$ and the maximum transfer rate is $100 \mathrm{k}$ events/s. The LUCRODs are able to count the number of pulses above a threshold (so-called hits), the pulse amplitude and the charge. The latter is obtained by dividing the pulse integral by the impedance of the electronics $(50 \mathrm{Ohm})$. Figure 2 shows a single photomultiplier pulse and illustrates how hits, amplitude and charge are defined.

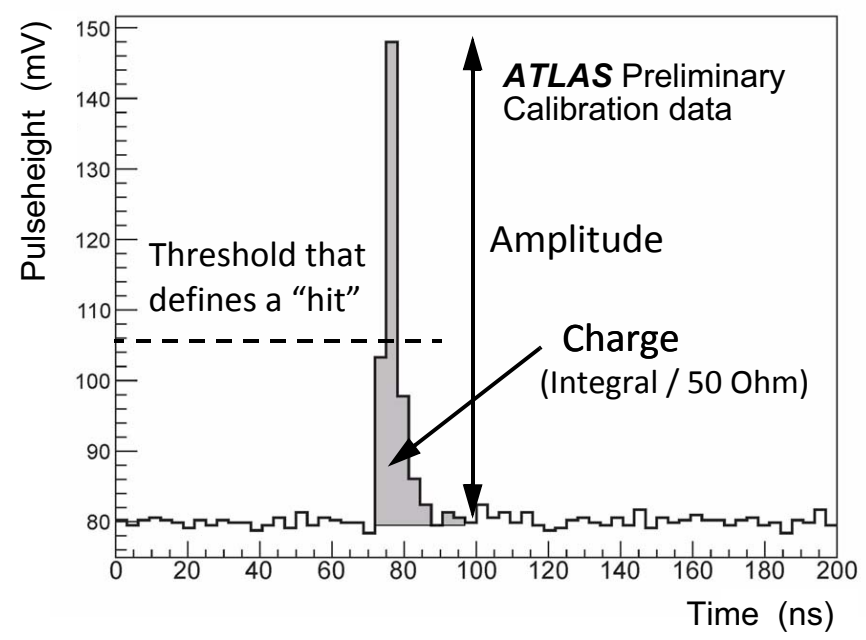

Figure 2. Typical shape of a digitised signal pulse with the LUCID-2 electronics [3].

Since the pulse width is smaller than $25 \mathrm{~ns}$, signals from individual LHC bunches can be separated.

The photomultipliers located in the proximity of the beamline are exposed to a large radiation flux. Radiation hardness tests performed with neutrons from reactors $\left(2.6 \times 10^{14} \mathrm{n} / \mathrm{cm}^{2}\right)$ and high-intensity gamma sources (200 kGray) showed no performance degradation.

\section{Detector stability monitoring}

One of the key requirements of a luminosity monitor is long term stability, therefore three different systems have been designed to monitor the gain of the LUCID-2 photomultipliers.

An innovative system consists in depositing drops of radioactive $\mathrm{Bi}-207$ on the photomultiplier quartz windows to produce electrons with energy above the Cherenkov threshold in the quartz. The photomultipliers can also be monitored with LED light transported via optical quartz fibers. The intensity of the LED source is monitored with PIN diodes to correct for eventual instabilities. A third monitoring system uses laser light from the ATLAS TILE calorimeter calibration system.

The advantage of using the $\mathrm{Bi}-207$ system is twofold. It does not have any components that can age or get damaged by radiation, such as LEDs, PIN diodes or optical fibers. In addition, Cherenkov light produced with $\mathrm{Bi}-207$ has similar wavelength and intensity compared to that produced by particles originating from pp collisions. Therefore, from 2017 on, all LUCID-2 subdetectors are monitored with $\mathrm{Bi}-207$ sources, with the exception of the fiber detector which is monitored with LED light. 
Runs with the LEDs and the Bi-207 systems were carried out between most LHC fills to measure mean amplitude and charge variations compared to the beginning of the year (see Figure 3 ).

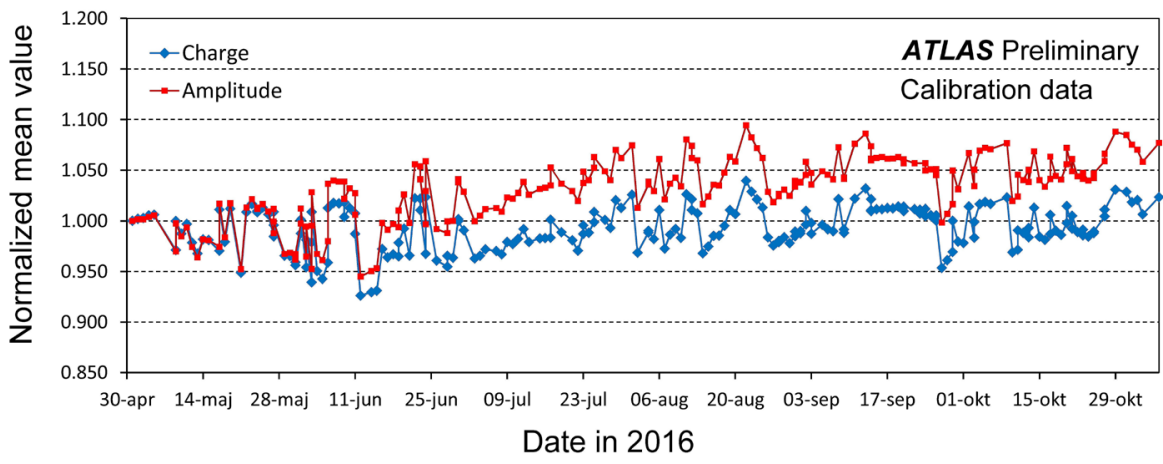

Figure 3. Average amplitude and charge measured in 2016 with a photomultiplier monitored with the Bi-207 system [3]. The measurements are normalised to the first one performed on the $1^{\text {th }}$ of May 2016.

The high voltage to the photomultipliers was automatically changed to compensate for the gain variation estimated from the associated mean amplitude variation. A rapid increase of the high voltage was necessary at the beginning of the data-taking period while typical corrections of a couple of volts were applied the rest of the year (see Figure 4).

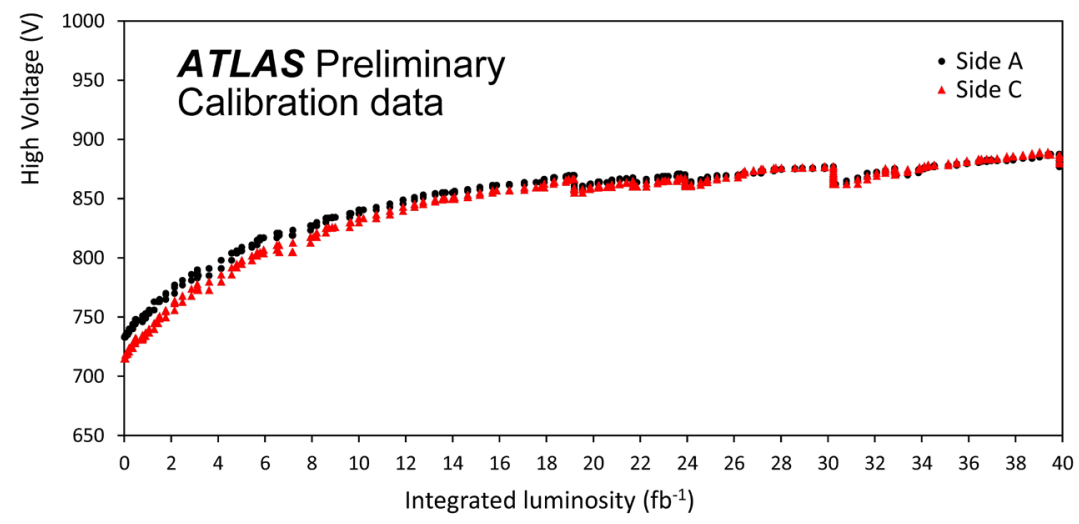

Figure 4. Average high voltage to 4 photomultipliers monitored with the Bi-207 system during 2016 as a function of the accumulated integrated luminosity [3]. The high voltage was automatically changed after each Bi-207 run in case of gain variations. The data are reported separately for the two LUCID modules (Side A and Side C).

\section{Luminosity measurement}

The LUCID-2 detector is the main online and offline luminosity monitor of the ATLAS experiment. It provides 104 different luminosity measurements for each of the LHC colliding bunches during an ATLAS luminosity block (LB), which is a time period of about 1 minute. 
LUCID measures the luminosity by counting the number of inelastic pp collisions in colliding bunches, which is assumed to be a Poissonian distribution with average $\mu$. The algorithms to measure $\mu$ are based on event counting, hit counting and charge counting, the difference between them being the raw quantity measured for each LB. In event counting one counts the number of bunch crossings with hits in the detectors, in hit counting the total number of hits is counted while in charge counting the total charge of the pulses is added up. The main algorithms in 2015 was based on event counting while in 2016 it was necessary to switch to hit counting due to larger $\mu$ values.

The measured $\mu$ value is called $\mu_{v i s}$ and depends on the efficiency of the counting method. The luminosity of a LB can be then expressed as:

$$
L_{L B}=\frac{f_{L H C}}{\sigma_{v i s}} \sum_{j=1}^{n_{b}} \mu_{j}^{v i s}
$$

where $f_{L H C}$ is the LHC revolution frequency, $n_{b}$ is the number of colliding bunches and $\sigma_{v i s}$ is the absolute luminosity calibration which is extracted from special LHC fills called van der Meer (vdM) fills [4]. In vdM fills, beams are separated in the horizontal (X) and vertical (Y) directions while the interaction rate is continuously measured with all algorithms. Absolute luminosity can be obtained from the width of the measured interaction rates in the $\mathrm{X}$ and $\mathrm{Y}$ directions, the peak $\mu_{v i s}$ values and the number of protons in the colliding bunches, the latter being measured with dedicated LHC detectors.

The statistical uncertainty in the LUCID measurement is negligible while the systematic effects are minimised by using the Bi-207 runs for offline corrections. The luminosity estimated from counting tracks in the inner detector is used to correct for pile-up effects, while those obtained from three different ATLAS calorimeter systems are used for comparisons (see Figure 5).

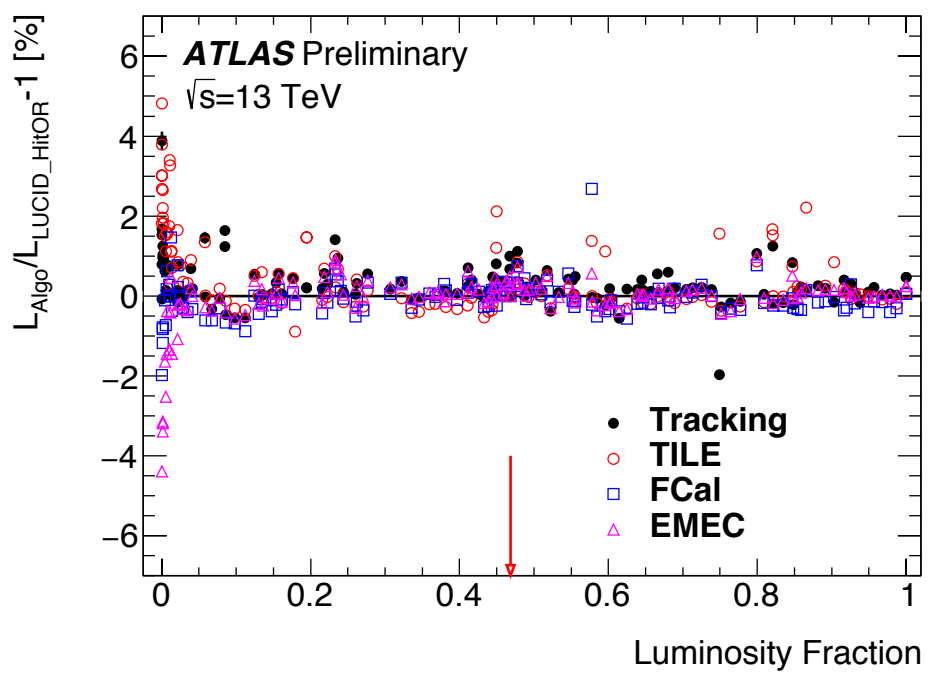

Figure 5. Fractional difference in run-integrated luminosity provided with the LUCID-2 detector, three ATLAS calorimeter systems (TILE, EMEC and FCAL) and the track-counting algorithm [5]. Each point corresponds to a different ATLAS run in 2016. All measurements are normalised to LUCID-2 in a run recorded on the $4^{\text {th }}$ of August (red arrow). The LUCID-2 measurements between the $1^{\text {st }}$ and the $8^{\text {th }}$ of July were reduced by $0.7 \%$ to compensate for the deviation from the track-counting algorithm. 
The total ATLAS luminosity uncertainty estimated in 2015 (2016) was 2.1\% (2.2\%).

\section{Summary}

The LUCID-2 luminosity monitor is a compact Cherenkov detector using the quartz windows of photomultipliers as the Cherenkov medium. Thanks to an innovative gain monitoring system based on Bi-207 radioactive sources, LUCID-2 is the main online and offline luminosity monitor of the ATLAS experiment during LHC Run 2. Combining the measurements of five ATLAS subdetectors, the luminosity has been estimated with a total uncertainty of $2.1 \%$ in 2015 and $2.2 \%$ in 2016 .

\section{References}

[1] ATLAS Collab. (G. Aad et al.), JINST 3, S08003 (2008).

[2] LUCID Collab. (G.L. Alberghi et al.), JINST 11, P05014 (2016).

[3] https://twiki.cern.ch/twiki/bin/view/AtlasPublic/ForwardDetPublicResults

[4] S. Van Der Meer, ISR-PO/68-31, KEK68-64.

[5] https://twiki.cern.ch/twiki/bin/view/AtlasPublic/LuminosityPublicResultsRun2 